\title{
A Spitzer IRAC Census of the Asymptotic Giant Branch Populations in Local Group Dwarfs. II. IC 1613
}

\author{
Dale C. Jackson, Evan D. Skillman, Robert D. Gehrz, Elisha Polomski, and Charles E. \\ Woodward \\ Astronomy Department, University of Minnesota, 116 Church St. S.E., Minneapolis, MN \\ 55419 \\ djackson@astro.umn.edu, skillman@astro.umn.edu, gehrz@astro.umn.edu, \\ elwood@astro.umn.edu, chelsea@astro.umn.edu
}

\begin{abstract}
We present Spitzer Space Telescope IRAC photometry of the Local Group dwarf irregular galaxy IC 1613. We compare our 3.6, 4.5, 5.8, and $8.0 \mu \mathrm{m}$ photometry with broadband optical photometry and find that the optical data do not detect $43 \%$ and misidentify an additional $11 \%$ of the total AGB population, likely because of extinction caused by circumstellar material. Further, we find that a narrowband optical carbon star study of IC 1613 detects $50 \%$ of the total AGB population and only considers $18 \%$ of this population in calculating the carbon to M-type AGB ratio. We derive an integrated mass-loss rate from the AGB stars of $0.2-1.0 \times 10^{-3} \mathrm{M}_{\odot} \mathrm{yr}^{-1}$ and find that the distribution of bolometric luminosities and mass-loss rates are consistent with those for other nearby metal-poor galaxies. Both the optical completeness fractions and mass-loss rates in IC 1613 are very similar to those in the Local Group dwarf irregular, WLM, which is expected given their similar characteristics and evolutionary histories.
\end{abstract}

Subject headings: stars: AGB - stars: carbon - stars: mass loss - galaxies : dwarf - galaxies : irregular - galaxies : Local Group - galaxies : individual(IC 1613)

\section{Introduction}

IC 1613 is an isolated member of the Local Group and serves as the prototype for the DDO Ir V type dwarf irregular (dI) galaxy (van den Bergh 2000a). Discovered by Wolf (1906), IC 1613's relative proximity (730 kpc; Dolphin et al. 2001), low inclination angle $\left(\mathrm{i}=38^{\circ}\right.$ Lake \& Skillman 1989), favorable galactic latitude $\left(\mathrm{b}=-60^{\circ}\right)$, and low foreground 
reddening $(\mathrm{E}(\mathrm{B}-\mathrm{V})=0.025 \mathrm{mag}$; Schlegel et al. 1998) make it an ideal target for stellar population and interstellar medium (ISM) studies. This is evidenced by the impressive number of previous studies of this galaxy, summarized by van den Bergh (2000a).

Lake \& Skillman (1989) studied the neutral hydrogen in IC 1613. They found the galaxy to be gas rich with the $\mathrm{H}$ I distribution being lumpy in the inner galaxy and smoother at larger radii. Cole et al. (1999) and Skillman et al. (2003) performed deep photometry of single HST/WFPC2 fields in the inner and outer galaxy, respectively. Both of these studies used high-precision photometry to derive star formation histories. They found continuous star formation over the past few hundred Myr, as well as populations of intermediate age and ancient stars.

IC 1613 is, in most respects, a typical irregular galaxy. Its metal abundance, current star formation rate, and dust content are all normal for a galaxy of its luminosity. The morphology consists of a prominent star forming region northeast of the galaxy center and a bar running linearly through the galaxy center from southeast to northwest. Perhaps the only exceptional property of IC 1613 is its nearly complete absence of star clusters (van den Bergh 1979; Wyder et al. 2000), which may be evidence of its isolated evolution (van den Bergh 2000b).

In this paper we present Part II of our Spitzer IRAC thermal infrared study of the stellar populations in Local Group dwarf irregulars with a census of the asymptotic giant branch stars in IC 1613. In \$2 we describe the observations, data reduction, and photometry. In $\$ 3$ we present the Spitzer IRAC images and describe the near- and mid-IR morphology of IC 1613. We characterize the infrared color-magnitude diagram (CMD) in \$4. In \$5 we detail a complete census of the asymptotic giant branch (AGB) stars, a comparison with broadband optical photometry (\$5.1), a comparison with an optical carbon star study (\$5.2), the determination of mass-loss rates (MLRs; \$5.3), and the total luminosity contribution from the AGB population (\$5.4). In $₫ 6$ we summarize our conclusions.

\section{Observations and Data Reduction}

\subsection{Infrared and Optical Data and Photometry}

Images of IC 1613 were obtained with the Infrared Array Camera (IRAC; Fazio et al. 2004) on board the Spitzer Space Telescope (Werner et al. 2004) in two separate AORs on 2003 December 21 UT and 2005 December 23 UT (AORKEYs: r5051648 and r15892736), respectively. Both AORs are part of a larger guaranteed time observing program (PID: 128, PI: R.D. Gehrz). Five individual $200 \mathrm{~s}$ frames at 4.5 and $8.0 \mu \mathrm{m}$ and 32 individual $30 \mathrm{~s}$ frames 
at 3.6 and $5.8 \mu \mathrm{m}$ were used resulting in total integration times of $1000 \mathrm{~s}$ per pointing at 4.5 and $8.0 \mu \mathrm{m}$ and $960 \mathrm{~s}$ per pointing at 3.6 and $5.8 \mu \mathrm{m}$. These data yielded $5-\sigma$ point source sensitivities of $4.5,3.5,10.7$, and $16.5 \mu \mathrm{Jy}$ at $3.6,4.5,5.8$, and $8.0 \mu \mathrm{m}$, respectively. Because the IRAC 3.6 and $5.8 \mu \mathrm{m}$ field of view is offset from that at 4.5 and $8.0 \mu \mathrm{m}$, a $3 \times 2$ mosaic in array coordinates approximately $15^{\prime} \times 10^{\prime}$ for each channel was created to map IC 1613 . This resulted in a coverage area with data in all four IRAC channels of approximately $9^{\prime} \times 9^{\prime}$ in size, centered at $\alpha(2000)=02: 06: 30, \delta(2000)=01: 04: 50$. Figure 1 shows this field of view overlaid on the Digitized Sky Survey image of IC 1613.

IRAC data (pipeline version 13.2.0) were reduced with the MOPEX1 reduction package, version 2006 March 1 . The backgrounds of individual frames were matched using the overlap routine. The mosaic program was used for outlier detection, image interpolation and co-addition, and to create the final mosaics. Very bright objects imaged in the program immediately preceding our observations resulted in persistent images in the $8.0 \mu \mathrm{m}$ data taken in 2003. These artifacts were effectively removed by subtracting a median-combined image of all of the dithered frames from each individual frame.

Point source photometry was done using the DAOPHOT II photometry package (Stetson 1987). A sharpness clip was applied to the photometry in each of the IRAC bands. This clip removes any objects from the photometry list whose radial profiles are significantly steeper or broader than the profile of the derived point spread function (PSF). We adopt the standard DAOPHOT errors as the total photometric errors for our sources. The IRAC instrument also has an estimated absolute calibration accuracy of $3 \%$ (Reach et al. 2005), although we do not include this uncertainty in our photometric errors.

Optical $V$ and $I$ photometry of IC 1613 were obtained from the OGLE-II microlensing survey (Udalski et al. 2001). These data are used to aid in identifying the stellar type of the stars detected in our IRAC data, which is challenging with IRAC data alone (see \$4). The optical data were acquired in the context of detecting objects that are variable (due to either microlensing events or intrinsic stellar variability) by taking a large number of frames, each with relatively short integration times. The photometry presented here is the mean of the individual photometric observations, including those objects found to be variable. The field of view of the Udalski et al. (2001) optical data covers the entire IRAC 3.6, 4.5, 5.8, and $8.0 \mu \mathrm{m}$ coverage area, except for a small triangular region $\sim 0.9$ square arcmin in size in the south-eastern edge of the IRAC coverage area (see Figure 1).

\footnotetext{
${ }^{1}$ MOPEX is available from the Spitzer Science Center at http://ssc.spitzer.caltech.edu/postbcd/
} 


\subsection{Foreground Star Contamination}

To estimate the number of foreground stars in both the optical and IR data we used the Milky Way stellar population synthesis model of Robin et al. (2003). Sources were modeled for the Galactic coordinates of IC 1613 in a one square degree field to reduce statistical error. The Robin et al. (2003) model provides magnitudes in the Johnson-Cousins L-band, whose central wavelength is close to that of the IRAC $3.6 \mu \mathrm{m}$ band. In the region of IC 1613 where we have coverage in the optical and all four IRAC channels, we expect 15 foreground stars in our IR color-magnitude diagram (CMD). These 15 foreground stars are expected to have $-7.5<\mathrm{M}_{3.6 \mu m}<-16$ and [3.6]-[4.5] colors near zero.

\section{Infrared Galaxy Morphology}

IRAC 3.6, 4.5, 5.8, and $8.0 \mu \mathrm{m}$ images of IC 1613 are shown in Figure 2, In general, the stars in these images are uniformly distributed throughout the images, consistent with

the regular elliptical distribution of red giants discussed by Baade \& Gaposchkin (1963), and dubbed Baade's Population II 'sheet' by Sandage (1971). In addition to the smooth distribution of stars, a concentration of bright stars is seen in the large star forming complex in the northeast. The only diffuse emission detected in these images is $8.0 \mu \mathrm{m}$ emission from hot $(\sim 350 \mathrm{~K})$ dust and/or polycyclic aromatic hydrocarbons. This emission is detected in regions roughly coincident with strong $\mathrm{H} \alpha$ emission (Jackson et al. 2006, and references therein).

\section{Comparison of Optical and IR Photometry}

The optical color-magnitude diagram (CMD) from the Udalski et al. (2001) data is shown in Figure 3, segregated into regions consisting of (a) blue objects that can include massive main sequence stars and unresolved H II regions, (b) asymptotic giant branch (AGB) stars, (c) red supergiants (RSGs), and (d) red giants (RGs) below the tip of the red giant branch (TRGB). We refer hereafter to the stars called out in (d) as sub-TRGB stars. These regions are used to identify the stellar populations in the infrared CMD, since IRAC data alone provide little information on stellar effective temperatures. These indicated regions are separated by gaps reflecting the estimated 1- $\sigma$ photometric error, which were employed to minimize misidentification of stellar types solely due to photometric errors. Reddening from circumstellar and interstellar material can also cause such misidentification. The optical CMD shows features of both recent and very old star formation, consistent with the finding 
of Skillman et al. (2003) that IC 1613 has a relatively constant star formation rate over the age of the Universe.

Figure 4 shows the $3.6 \mu \mathrm{m}$ absolute magnitude versus [3.6]-[4.5] $\mu \mathrm{m}$ CMD. The left panel shows all of the stars detected at both 3.6 and $4.5 \mu \mathrm{m}$, with photometric errors, averaged over one magnitude bins, displayed. The left panel also shows the $50 \%$ completeness limit (black line) derived from artificial star tests. The right panel shows the stars detected in $V, I$, and at 3.6 and $4.5 \mu \mathrm{m}$, with RSGs shown as black stars, AGB stars as green triangles, red giants as red squares, and blue objects as blue circles. The right panel contains a vector showing the displacement a star would experience due to 10 visual magnitudes of extinction and reddening (Indebetouw et al. 2005; Rieke \& Lebofskv 1985), though this vector does not include the effects of dust emission, which can be significant in the mid-IR. The right panel also shows the AGB limit, which was determined by taking the Groenewegen (2006) models for carbon-rich AGB stars with $\mathrm{T}_{\text {eff }}=2650$ and $\mathrm{T}_{\text {eff }}=3600$ and negligible mass-loss and scaling them to an absolute bolometric magnitude of $\mathrm{M}_{b o l}=-7.1$. The AGB limit depicted in this figure is a line connecting these two models. AGB stars that are losing significant mass can be brighter than this limit due to thermal emission of the mass-loss material increasing their $3.6 \mu \mathrm{m}$ flux (as shown in detail in \$5.3), however, any such objects would also have red [3.6]-[4.5] colors. None of these objects are observed, thus, we conclude all of the stars brighter than the AGB limit are true red supergiants.

The right panel also shows the $3.6 \mu \mathrm{m}$ TRGB. The value of the TRGB we adopt $\left(\mathrm{M}_{3.6}=-6.2 \pm 0.2\right)$ was determined by inspecting the $3.6 \mu \mathrm{m}$ luminosity function for sources detected at both 3.6 and $4.5 \mu \mathrm{m}$. The $3.6 \mu \mathrm{m}$ luminosity function (the number of stars in each 0.2 magnitude bin at $3.6 \mu \mathrm{m}$ ) is shown in Figure 5. We adopt this value of the TRGB based on the abrupt drop in detections at that magnitude. Also, optically classified sub-TRGB red giants with blue [3.6]-[4.5] colors are observed with $\mathrm{M}_{3.6}$ magnitudes up to this value, but above this value only optically classified sub-TRGB red giants with very red colors are observed. The IR fluxes of these objects are consistent with mass-losing AGBs (see \$5) rather than sub-TRGB red giants, supporting our adopted value of $\mathrm{M}_{3.6}=-6.2$ for the $3.6 \mu \mathrm{m}$ TRGB. This value is 0.2 magnitudes fainter than the value found for the Large Magellanic Cloud (LMC) $\left(\mathrm{M}_{L^{\prime}}=-6.4\right.$; van Loon et al. 2005) and 0.4 magnitudes lower than that adopted for WLM (Jackson et al. 2007). It is unlikely that the difference in values of the TRGB between IC 1613 and WLM are actually so disparate, given their similar metallicities. The difference in these values likely reflects the uncertainty in our adopted TRGB values. This uncertainty is not a major concern, however, because shifting the value of the TRGB by up to 0.5 magnitudes affects the detection statistics only slightly (see $\$ 5.1$ ).

In general, the $\mathrm{M}_{3.6}$ versus [3.6]-[4.5] CMD of IC 1613 is very similar to that of WLM, 
with the only major difference being that there are significantly more sub-TRGB red giants detected in IC 1613 than in WLM because WLM is more distant. As is the case of WLM, a small population of stars redward of the main stellar distribution is detected, though fewer are seen in IC 1613 than in WLM. As we discuss in \$5.3, the infrared fluxes and colors of these objects are consistent with mass-losing AGB stars.

Figure 6] is the $\mathrm{M}_{8.0}$ versus [3.6]-[8.0] CMD for IC 1613. This CMD shows a narrow vertical feature with $[3.6]-[8.0] \sim 0$ and another broad distribution of red objects with $-12<\mathrm{M}_{8.0}<-7.51<[3.6]-[8.0]<4$. These red objects are also the most luminous, reddest objects in the $\mathrm{M}_{3.6}$ versus [3.6]-[4.5] CMD, with typical colors between 0.5 and 1.0.

Figure 7 shows the spatial distributions of different stellar types based on their optical and IR fluxes. The distributions of both red giants and AGB stars are very smooth with no obvious concentrations aside from the radial stellar gradient. A thin gap is observed in the optical data, which is certainly an instrumental effect, though this is not mentioned in Udalski et al. (2001). There are two conspicuous features in the blue objects, which outline the young stellar distribution; a bar running across the midplane of the galaxy and a large clump in the northeast, which highlights the most active star forming complex in IC 1613. It is clear from this figure that neither the optical nor the IR data presented here reach the outermost regions of the stellar population, as the stellar density is still dropping off at the edges of the images. In the analogous plot in Jackson et al. (2007), the effects of crowding could clearly be seen in WLM as clumps of bright, young stars coincided spatially with an absence of fainter red giants. In IC 1613 we see no evidence for such an effect, likely because

IC 1613 is seen much more face on $\left(i=38^{\circ}\right.$, Lake \& Skillman 1989) than WLM $\left(i=69^{\circ}\right.$, Jackson et al. 2004).

In Table 2 we present the detection statistics for the optical and infrared photometry of IC 1613. We detect 5211, 3162, 886, and 618 point sources at $3.6,4.5,5.8$, and $8.0 \mu \mathrm{m}$, respectively, and the Udalski et al. (2001) $V$ and $I$ data contain 7266 point sources within the optical and IR coverage region. We detect 183 point sources in all four IRAC channels with no corresponding detection in the optical. Three of these are RSGs (i.e., they are above the AGB limit), two of these are very red objects below the TRGB, and the remaining 178 are either RSGs, AGB stars, or blue objects.

\section{The AGB Stars}

As shown in Figure 4, our completeness limit is more than 0.5 mag fainter than the TRGB for the bluest objects and $1.5 \mathrm{mag}$ fainter than the TRGB for red objects. Con- 
sequently, our data constitutes a complete census of the super-TRGB AGB stars with the exception of a very small number of objects not detected due to crowding.

As described in Jackson et al. (2007), though our data should represent a complete census of the AGB stars, we cannot distinguish between AGB stars, RSGs, and blue objects using IRAC data alone. However, above the TRGB, optically identified RSGs and blue objects comprise only $9 \%$ (95 out of 1041) of the objects between the TRGB and the AGB limit. Therefore, we assume any object between the TRGB and the AGB limit is an AGB star unless its optical identification dictates otherwise, with the understanding that there may be a small amount of contamination by other stellar types.

\subsection{Optical Completeness}

Altogether we detect 1052 AGB stars in our IRAC data. Of these $57 \%$ are detected in the Udalski et al. (2001) $V$ and $I$ data. In addition to the $43 \%$ of AGB stars detected in the IR but not seen in the optical, $11 \%$ of the IR detected AGB stars are located in the optical CMD where they would be misidentified as sub-TRGB red giants.

For comparison, in WLM Jackson et al. (2007) found $39 \%$ of the IR detected AGB stars were not seen in the optical data of Massey et al. (2006), and an additional $4 \%$ were optically detected, but misidentified as sub-TRGB red giants. An important check of our AGB statistics for WLM was recently provided by Valcheva et al. (2007), who performed $\mathrm{J}$ and $\mathrm{K}_{S}$ near-IR photometry. Altogether they detect 355 AGB stars (carbon + M-type AGBs), in comparison with the 331 AGB stars we detect in the same field of view. It is not surprising that the number of AGBs we detect in WLM and IC 1613 are in such close agreement given the striking similarities in the evolution, and particularly in the intermediate age star formation histories, of these galaxies (Figures 25 and 35 in Dolphin et al. 2005).

In Figure 8 we show the fraction of optical completeness as a function of [3.6]-[4.5] color (left panel) and as a function of the modeled AGB wind optical depth (right panel; AGB mass-loss modeling is described in detail in \$5.3). This figure shows the optical completeness fraction is a smoothly declining function of [3.6]-[4.5] color, as is expected if the incompleteness is due to extinction by circumstellar material. Much like in the case of WLM, we see the completeness fraction fall to $0 \%$ at a $[3.6]-[4.5]$ color of $\sim 0.9$.

It is important to understand to what degree our adopted value of the TRGB affects these completeness statistics. By shifting our adopted value 0.5 mag fainter the fraction of optically undetected AGB stars changes by only $2 \%$, while shifting the TRGB 0.5 mag brighter would increase the optically undetected AGB fraction by $10 \%$. As in the case 
of WLM, this increase in optical incompleteness with increasing luminosity supports our conclusion that the optically undetected AGB stars are not seen because they are enshrouded by circumstellar material, since the mass-loss phase in AGB stars occurs somewhat above the TRGB and should consequently be more conspicuous at higher luminosities.

\subsection{The Carbon Stars}

Narrowband and broadband optical photometry of IC 1613 using the CN/TiO technique was performed by Albert et al. (2000). In comparison with our IRAC photometry, the Albert et al. (2000) data detected $50 \%$ of the total AGB population seen in our IRAC data. This value is slightly lower than the fraction detected by Udalski et al. (2001) and is caused by the brighter completeness limits of the narrowband data, since the broadband data from Albert et al. (2000) is significantly more sensitive than that of Udalski et al. (2001).

Albert et al. (2000) distinguish between oxygen- and carbon-rich AGBs using a colorcolor diagram. At relatively red $(R-I)$ colors oxygen- and carbon-rich AGBs have easily distinguishable CN-TiO colors, however, AGBs with bluer $(R-I)$ colors are not easily separated. Consequently, a color limit is imposed, redward of which the discrimination of chemical composition can be made. Within our IRAC coverage area Albert et al. (2000) identified 74 carbon stars. Assuming their C/M ratio of 0.64, this yields 190 total AGB stars (C + M-type). Thus, after applying their imposed color limit, $18 \%$ of the total AGB population was considered for the $\mathrm{C} / \mathrm{M}$ ratio.

Figure 9 shows our IRAC photometry of the carbon stars identified by Albert et al. (2000). The carbon stars have relatively blue colors, probably due to the low-metallicity, and consequently the low optical depth of circumstellar wind material of AGBs in IC 1613, though four red carbon stars are seen that are likely losing significant mass. There are also three carbon stars identified by Albert et al. (2000) that we find to be well below the TRGB at $3.6 \mu \mathrm{m}$. These three stars are also below the TRGB in the optical, and are possibly extrinsic carbon stars. Because it is very unlikely that these three objects are in fact true carbon-rich AGB stars we do not consider them in any of the carbon star statistics. With the exception of these three objects, the carbon stars identified by Albert et al. (2000) have relatively bright $3.6 \mu \mathrm{m}$ luminosities compared to the rest of the AGB stars.

The comparison between our IR photometry and the narrowband optical data in IC 1613 is nearly identical to the values we found for this same comparison in WLM (Jackson et al. 2007). In WLM we found the completeness fraction of the Battinelli \& Demers (2004) optical narrowband data was $63 \%$ of the total AGB population observed in our IRAC data and the 
$\mathrm{C} / \mathrm{M}$ ratio was based on $18 \%$ of the IRAC detected AGB stars. Like in the comparison of broadband optical completeness, the striking similarity between AGB populations in IC 1613 and WLM is likely due to their similar star formation histories and metallicity evolution.

In Jackson et al. (2007) we stated that the Battinelli \& Demers (2004) optical CN/TiO study of WLM detected only $18 \%$ of the total AGB population, which is misleading. Again, because of the necessity to impose a color limit to discriminate between carbon- and oxygenrich $\mathrm{AGBs}$, relatively blue $\mathrm{AGB}$ stars are ignored in the calculation of the $\mathrm{C} / \mathrm{M}$ ratio. In WLM $18 \%$ of the total AGB population was used to calculate the $\mathrm{C} / \mathrm{M}$ ratio, while an additional $45 \%$ of the total population was detected but not used in the $\mathrm{C} / \mathrm{M}$ ratio because the chemical composition could not be determined. This point is critical to consider when comparing $\mathrm{C} / \mathrm{M}$ ratios obtained using different methods. As an example, recent near-IR photometry of WLM (Valcheva et al. 2007) found a C/M ratio of 0.58 (corrected for foreground contamination). This is in sharp contrast with the Battinelli \& Demers (2004) value of 12.4, though these values must not be directly compared because, as is shown here, the Battinelli \& Demers (2004) C/M ratio was calculated using 18\% of the AGB stars, while the (Valcheva et al. 2007) value considered the entire AGB population.

\subsection{Mass-Loss Rates}

The mass-loss rates (MLRs) of AGB stars in IC 1613 were determined by comparing their infrared fluxes with the radiative transfer models of Groenewegen (2006). The [3.6]-[4.5] color of AGB stars corresponds to a unique wind optical depth $(\tau)$, given assumptions about the wind composition and stellar effective temperature $\left(\mathrm{T}_{\text {eff }}\right)$, as we discuss below. The [3.6]-[4.5] colors were linearly interpolated onto the Groenewegen (2006) models to estimate $\tau$, then scaled according to the van Loon (2000) MLR prescription, where: $\dot{M} \propto \tau \psi^{-\frac{1}{2}} \mathrm{~L}^{\frac{3}{4}}, \psi$ is the dust-to-gas ratio, and L is the stellar luminosity. For IC 1613 we adopt the nebular oxygen abundance of $12+\log (\mathrm{O} / \mathrm{H})=7.62$ (Lee et al. 2003), which corresponds to a metallicity of $0.9 \% \mathrm{Z}_{\odot}$. Given that the dust-to-gas ratio scales as $\psi=\psi_{\odot} 10^{-[\mathrm{Fe} / \mathrm{H}]}$ and $\psi_{\odot}=0.005$ (van Loon et al. 2005), we adopt $\psi_{I C 1613}=4.8 \times 10^{-4}$.

It is more appropriate to apply the metallicity of IC 1613 as it was a few Gyr ago when the current AGB population was forming, rather than the current metallicity. However, determining this value empirically is difficult, and Skillman et al. (2003) find very little metallicity evolution in IC 1613 over the past few Gyr, so applying the current metallicity to the AGB stars should be reasonably accurate.

There are two major uncertainties in deriving MLRs for AGB stars using only our 3.6 
and 4.5 micron data. The first is that there is a degeneracy between $\tau$ and $\mathrm{T}_{\text {eff }}$, such that cool AGB stars losing no mass can have the same [3.6]-[4.5] color as warmer AGBs that are losing mass. Because of this degeneracy we are forced to derive MLRs by adopting a single $\mathrm{T}_{\text {eff }}$ for all of the AGB stars, which is certainly not physical. As a consequence, the MLRs we find for individual blue AGB stars (i.e., AGB stars with [3.6]-[4.5]<0.5) should not be overinterpreted. Marengo et al. (2006) showed that atmospheric molecular absorption features can also effect the IRAC colors of AGB stars, introducing additional uncertainty to these measurements, although these effects have not yet been quantified. However, as emphasized in Jackson et al. (2007), because the majority of the integrated mass-loss for IC 1613 comes from sources much redder than [3.6]-[4.5] > 0.5, where the MLR we calculate is much less sensitive to $\mathrm{T}_{\text {eff }}$, the integrated MLR for the galaxy as well as those found for red AGB stars are robust.

The second major uncertainty in our MLR calculation is that we have no a priori information about the chemical composition of the AGB winds, and therefore, must assume a single chemical composition for the entire AGB population. This assumption is also unphysical, and the disparity between the integrated MLRs we measure for all of the modeled wind compositions and effective temperatures differ by a factor of four.

Figure 10 shows the MLR versus bolometric luminosity for each AGB and RSG in IC 1613 assuming a wind composition of 85\% amorphos carbon (AMC) $+15 \% \mathrm{SiC}$ and effective temperatures of $\mathrm{T}_{\text {eff }}=2650 \mathrm{~K}$ (filled circles) and $\mathrm{T}_{\text {eff }}=3600 \mathrm{~K}$ (open circles). Again, we consider any object detected at 3.6 and $4.5 \mu \mathrm{m}$ that is above the TRGB at 3.6 $\mu \mathrm{m}$ to be an AGB star, except for those optically classified as blue objects, since unresolved $\mathrm{H}$ II regions lie in the same part of the IR CMD as mass-losing AGBs. In comparison with the tracks of increasing wind optical depth (see Groenewegen 2006; Jackson et al. 2007), this plot shows that the most heavily mass-losing stars are among the most luminous AGBs. The maximum MLRs observed in IC 1613 are in very good agreement with the maximum MLRs seen in WLM (Jackson et al. 2007) and the LMC (van Loon et al. 1999), and somewhat higher than the classic single-scattering MLR limit predicted by Jura (1984). We also see a number of RSGs above the AGB limit, none of which show infrared excess.

The total MLRs we derive for IC 1613 are $2.4 \times 10^{-4} \mathrm{M}_{\odot} \mathrm{yr}^{-1}$ for $\mathrm{T}_{\text {eff }}=2650 \mathrm{~K}$ and $85 \% \mathrm{AMC}+15 \% \mathrm{SiC}, 4.3 \times 10^{-4}$ for $\mathrm{T}_{\text {eff }}=3600 \mathrm{~K}$ and $85 \% \mathrm{AMC}+15 \% \mathrm{SiC}, 1.0 \times 10^{-3}$ $\mathrm{M}_{\odot} \mathrm{yr}^{-1}$ for $\mathrm{T}_{\text {eff }}=2500 \mathrm{~K}$ and $60 \%$ Silicate $+40 \%$ Aluminum Oxide, and $8.8 \times 10^{-4}$ for $\mathrm{T}_{\text {eff }}=2500$ and $100 \%$ Silicate. These values are approximately a factor of two lower than those found for WLM. Considering that a significant fraction of the mass-loss is coming from a small number of objects with short mass-loss timescales, the integrated MLR of a relatively low-mass dwarf like IC 1613 could be highly time-variable and the consistency between the 
MLRs of WLM and IC 1613 is notable.

\subsection{Luminosity Contribution From AGB Stars}

For point sources detected at 3.6 and $4.5 \mu \mathrm{m}$ we find a total $3.6 \mu \mathrm{m}$ flux of $72.4 \mathrm{mJy}$. $85 \%$ of this total flux is from AGB stars and RSGs, which is very similar to the case of WLM, where we found $79 \%$ of the flux in point sources was from sources brighter than the TRGB. The total flux in point sources we measure for IC 1613 constitutes $79 \%$ of the integrated 4.5 $\mu \mathrm{m}$ flux detected by Lee et al. (2006).

Following van Loon et al. (2005) and adopting an age of the current super-TRGB stellar population of 2 Gyr we find a total stellar mass of $1.7 \times 10^{7} \mathrm{M}_{\odot}$. This value is a factor of 2.6 higher than the $6.6 \times 10^{6} \mathrm{M}_{\odot}$ estimated by Lee et al. (2006). Because the van Loon et al. (2005) method of measuring stellar mass assumes a single-aged stellar population and is dependent on the age of that population, our stellar mass may be over-estimated since a number of stars above the TRGB in IC 1613 are likely RSGs younger than 2 Gyr old.

\section{Summary of Results and Conclusions}

We present Spitzer Space Telescope thermal near-IR photometry of IC 1613. These data are compared with the optical $V$ and $I$ photometry of Udalski et al. (2001) and we find that $43 \%$ of the IR detected AGB stars are not detected in the optical and an additional $11 \%$ of the AGBs are misidentified as sub-TRGB red giants. We show that the optical incompleteness fraction is very well correlated with the [3.6]-[4.5] color, which indicates that the optically undetected AGB stars have been reddened by circumstellar material beyond the optical completeness limits.

Our IR photometry is also compared with the narrowband optical carbon star study of Albert et al. (2000) and we find their study detects $50 \%$ of the total AGB population, and their calculated $\mathrm{C} / \mathrm{M}$ ratio is based on $18 \%$ of this population. Further, the number of IR detected AGB stars we find for WLM in Jackson et al. (2007) is in excellent agreement with the recent near-IR study of Valcheva et al. (2007), who find a C/M ratio of 0.58.

We find the total MLRs from AGB stars to be $0.2-1.0 \times 10^{-3} \mathrm{M}_{\odot} \mathrm{yr}^{-1}$, depending on the assumed effective temperatures and chemical composition of the mass-loss winds of the AGB stars. The distribution of bolometric luminosities and MLRs of individual AGB stars is in excellent agreement with those observed in the Magellanic Clouds (van Loon et al. 1999; van Loon 2006). 
We show that the dominant contribution (85\%) to the thermal near-IR luminosity of IC 1613 is from the AGBs and RSGs.

Finally, the properties of the AGB populations in IC 1613 (i.e., the broadband and narrowband optical completeness fractions and MLRs) are nearly identical to those found in Jackson et al. (2007) for WLM. This is no doubt due to their similar galaxy characteristics and evolutionary histories, and is likely representative of star-forming dIs.

We sincerely thank Serge Demers for providing his narrowband photometry of WLM and IC 1613 and for extremely valuable comments regarding narrowband optical completeness statistics. We also thank the anonymous referee for their careful reading of the manuscript and their valuable comments, particularly regarding the possible detection of extrinsic carbon stars. This work is based in part on observations made with the Spitzer Space Telescope, which is operated by the Jet Propulsion Laboratory, California Institute of Technology under NASA contract 1407. Support for this work was provided by NASA through Contract Numbers 1256406 and 1215746 issued by JPL/Caltech to the University of Minnesota. This research has made use of NASA's Astrophysics Data System Bibliographic Services and the NASA/IPAC Extragalactic Database (NED) which is operated by the Jet Propulsion Laboratory, California Institute of Technology, under contract with the National Aeronautics and Space Administration.

\section{REFERENCES}

Albert, L., Demers, S., \& Kunkel, W. E. 2000, AJ, 119, 2780

Baade, W., \& Gaposchkin, C. H. P. 1963, Evolution of stars and galaxies (Cambridge: Harvard University Press)

Battinelli, P., \& Demers, S. 2004, A\&A, 416, 111

Cole, A. A., et al. 1999, AJ, 118, 1657

Dolphin, A. E., et al. 2001, ApJ, 550, 554

Dolphin, A. E., , Weisz, D. R., Skillman, E. D., \& Holtzman, J. A. 2005, preprint (astro-ph/0506430)

Fazio, G. G., et al. 2004, ApJS, 154, 10

Groenewegen, M. A. T. 2006, A\&A, 448, 181 
Indebetouw, R., et al. 2005, ApJ, 619, 931

Jackson, D. C., Skillman, E. D., Cannon, J. M., \& Côté, S. 2004, AJ, 128, 1219

Jackson, D. C., Cannon, J. M., Skillman, E. D., Lee, H., Gehrz, R. D., Woodward, C. E., \& Polomski, E. 2006, ApJ, 646, 192

Jackson, D. C., Skillman, E. D., Gehrz, R. D., Woodward, C. E., \& Polomski, E. 2007, ApJ, 656, 818

Jura, M. 1984, ApJ, 282, 200

Lake, G., \& Skillman, E. D. 1989, AJ, 98, 1274

Lee, H., Grebel, E. K., \& Hodge, P. W. 2003, A\&A, 401, 141

Lee, H., Skillman, E. D., Cannon, J. M., Jackson, D. C., Gehrz, R. D., Polomski, E. F., \& Woodward, C. E. 2006, ApJ, 647, 970

Marengo, M., Hora, J. L., Barmby, P., Willner, S. P., Allen, L. E., Schuster, M. T., \& Fazio, G. G. 2006, preprint astro-ph/0611346)

Massey, P., Olsen, K. A. G., Hodge, P. W., Strong, S. B., Jacoby, G. H., Schlingman, W., \& Smith, R. C. 2006, AJ, 131, 2478

Reach, W. T., et al. 2005, PASP, 117, 978

Rieke, G. H., \& Lebofsky, M. J. 1985, ApJ, 288, 618

Robin, A. C., Reylé, C., Derrière, S., \& Picaud, S. 2003, A\&A, 409, 523

Sandage, A. 1971, ApJ, 166, 13

Schlegel, D. J., Finkbeiner, D. P., \& Davis, M. 1998, ApJ, 500, 525

Skillman, E. D., Tolstoy, E., Cole, A. A., Dolphin, A. E., Saha, A., Gallagher, J. S., DohmPalmer, R. C., \& Mateo, M. 2003, ApJ, 596, 253

Stetson, P. B. 1987, PASP, 99, 191

Udalski, A., Wyrzykowski, L., Pietrzynski, G., Szewczyk, O., Szymanski, M., Kubiak, M., Soszynski, I., \& Zebrun, K. 2001, Acta Astronomica, 51, 221

Valcheva, A. T., Ivanov, V. D., Ovcharov, E. P., \& Nedialkov, P. L. 2007, ArXiv Astrophysics e-prints, arXiv:astro-ph/0701727 
van den Bergh, S. 1979, ApJ, 230, 95

van den Bergh, S. 2000, The galaxies of the Local Group (Cambridge, UK: Cambridge University Press)

van den Bergh, S. 2000, PASP, 112, 932

van Loon, J. T. 2006, ASP Conf. Ser. 353: Stellar Evolution at Low Metallicity: Mass Loss, Explosions, Cosmology, 353, 211

van Loon, J. T., Marshall, J. R., \& Zijlstra, A. A. 2005, A\&A, 442, 597

van Loon, J. T. 2000, A\&A, 354, 125

van Loon, J. T., Groenewegen, M. A. T., de Koter, A., Trams, N. R., Waters, L. B. F. M., Zijlstra, A. A., Whitelock, P. A., \& Loup, C. 1999, A\&A, 351, 559

Volders, L. M. J. S., \& Högbom, J. A. 1961, Bull. Astron. Inst. Netherlands, 15, 307

Werner, M. W., et al. 2004, ApJS, 154, 1

Wolf, M. 1906, MNRAS, 67, 91

Wyder, T. K., Hodge, P. W., \& Cole, A. 2000, PASP, 112, 594 
Table 1. Basic Properties of IC 1613

\begin{tabular}{lcc}
\hline \hline \multicolumn{1}{c}{ Quantity } & Value & Reference \\
\hline Right Ascension, $\alpha(2000)$ & 010446.4 & 1 \\
Declination, $\delta(2000)$ & +020845.9 & 1 \\
Heliocentric velocity, $\mathrm{V}_{\odot}\left(\mathrm{km} \mathrm{s}^{-1}\right)$ & $-232 \mathrm{~km} \mathrm{~s}^{-1}$ & 1 \\
Distance, D (Mpc) & $0.73 \pm 0.02$ & 2 \\
Morphological Type & $\mathrm{Ir} \mathrm{V}$ & 3 \\
$12+\log (\mathrm{O} / \mathrm{H})$ & 7.62 & 4 \\
Total H I mass $\left(\mathrm{M}_{\odot}\right)$ & $6.5 \times 10^{7}$ & 5 \\
Inclination angle $($ degrees $)$ & 38 & 1 \\
Position angle (degrees) & 58 & 1 \\
Rotational velocity $\left(\mathrm{km} \mathrm{s}{ }^{-1}\right)$ & 25 & 1 \\
Conversion factor $(\mathrm{pc} / \mathrm{arcmin})$ & 212 & 2 \\
\hline
\end{tabular}

References. - (1) Lake \& Skillman (1989). (2) Dolphin et al. (2001). (3) van den Bergh (2000a). (4) Lee et al. (2003). (5) Volders \& Högbom (1961). 
Table 2. Detection Statistics

\begin{tabular}{lc}
\hline \hline \multicolumn{2}{c}{ Total Point Source Detections in All Wavelengths } \\
\hline Filter & Number \\
\hline Both V and I & 7266 \\
$3.6 \mu \mathrm{m}$ & 5211 \\
$4.5 \mu \mathrm{m}$ & 3162 \\
$5.8 \mu \mathrm{m}$ & 886 \\
$8.0 \mu \mathrm{m}$ & 618 \\
& \\
\multicolumn{1}{c}{$\quad$ Detections in All Four IRAC bands, But Not V and I } \\
\hline Object type & Number \\
\hline Total & 183 \\
RSG (Above the AGB limit) & 3 \\
AGB/RSG (Above the TRGB, below the AGB limit) & 178 \\
Below the TRGB & 2 \\
\multicolumn{1}{c}{$3.6 \mu \mathrm{m}$ Point Source Flux (Total Flux = 72.4 mJy) } \\
\\
\hline Object type & Fraction \\
\hline Brighter than the TRGB & $85 \%$ \\
Fainter than the TRGB & $15 \%$ \\
$\quad \quad$ Optical Detection Fractions of IR Identified AGBs \\
\hline Filter & Fraction \\
\hline V and I & $57 \%$ \\
Detected but misidentified in V and I & $11 \%$ \\
Narrow-band optical & $18 \%$ \\
\hline
\end{tabular}




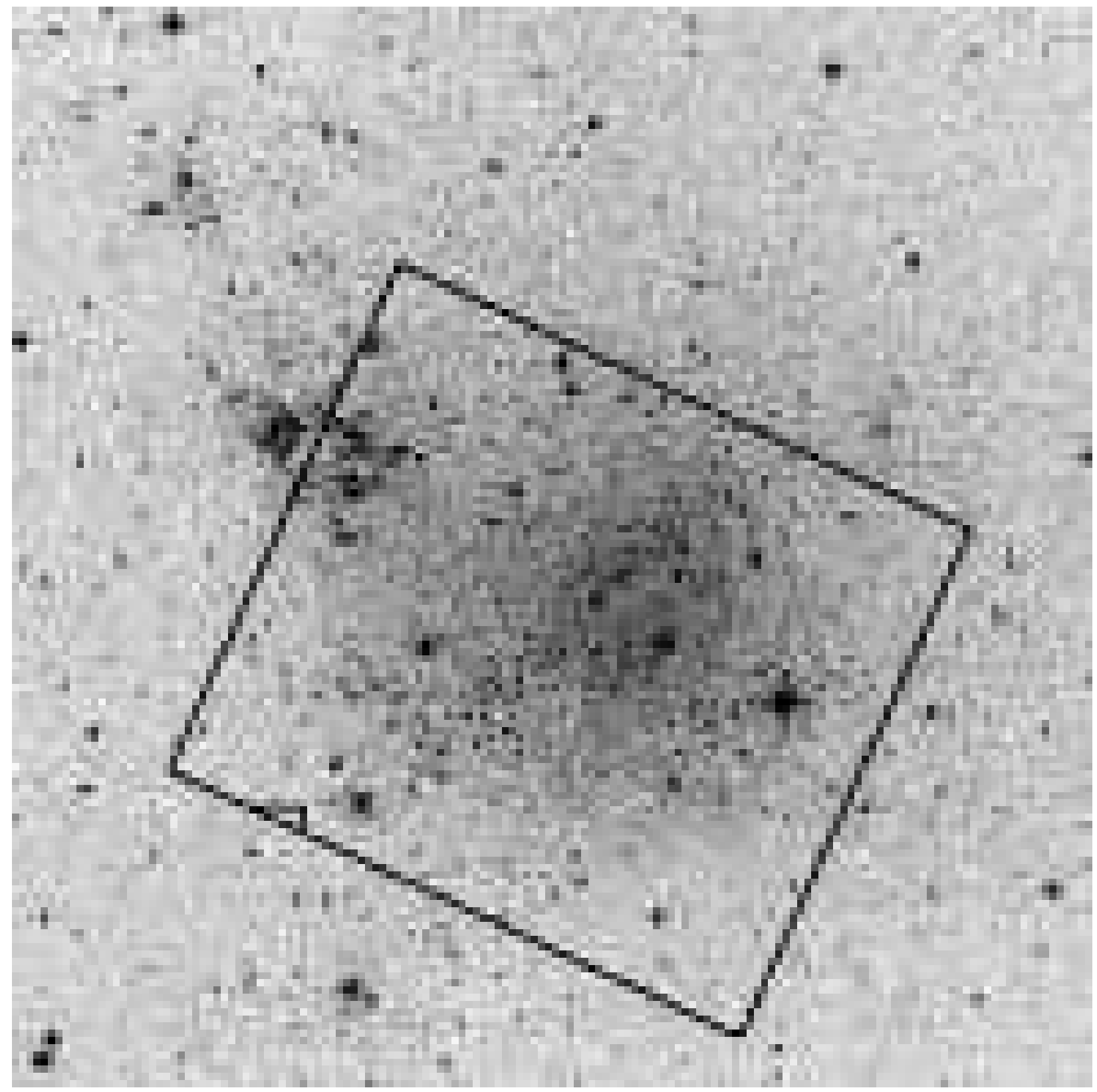

Fig. 1.- Digitized Sky Survey image of IC 1613, $14^{\prime} \times 14^{\prime}$ in size. The region of sky with coverage in all four IRAC bands is overlaid in black, and the small triangular region with no optical $V$ and $I$ coverage is shown in the southeast. North is up and East is left. 


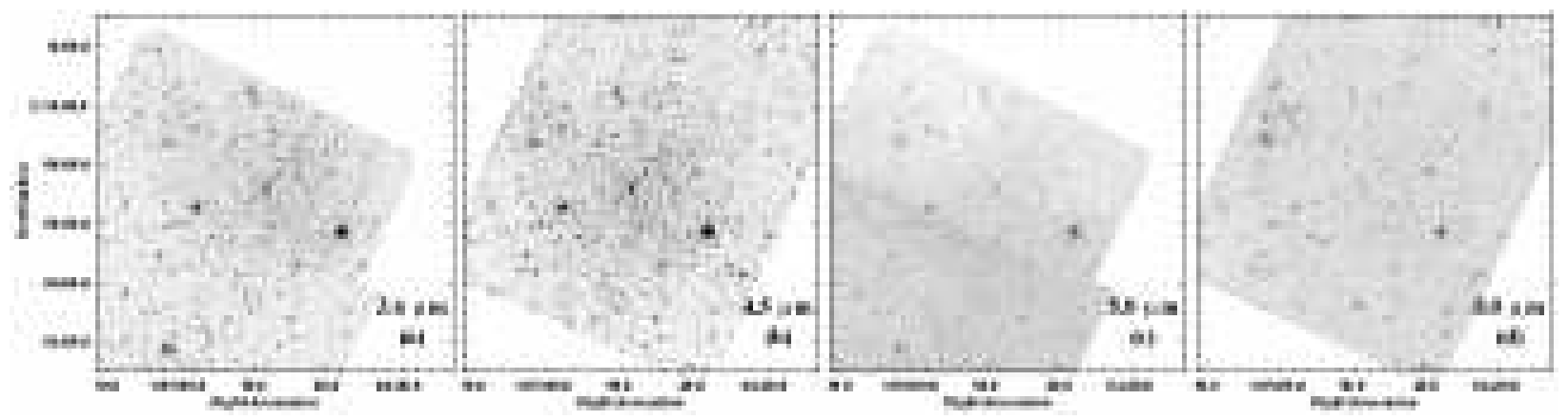

Fig. 2.- IRAC 3.6, 4.5, 5.8, and $8.0 \mu \mathrm{m}$ images of IC 1613. North is up and East is left. 


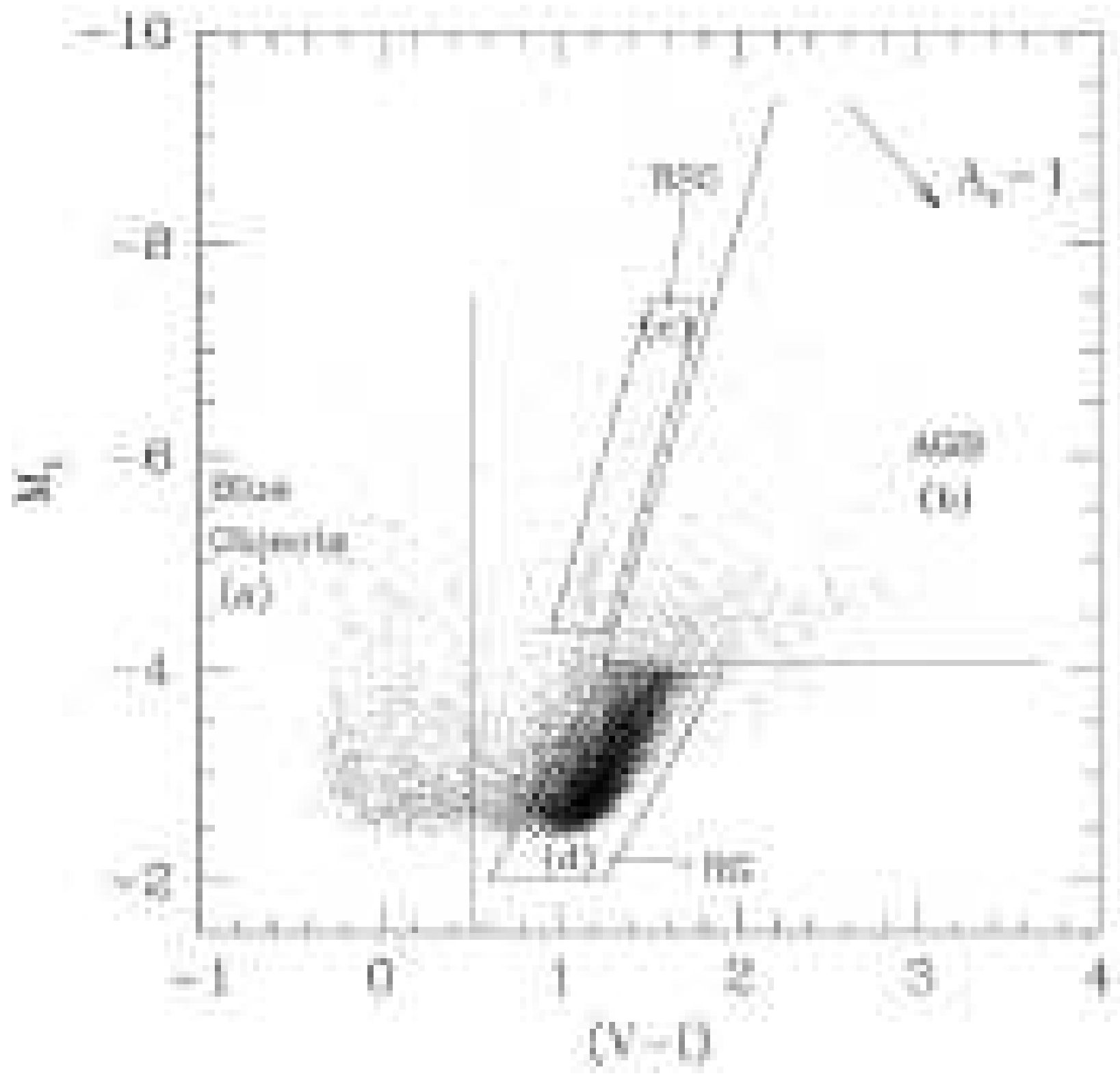

Fig. 3.- Absolute I versus $V-I$ color magnitude diagram of IC 1613 using data from Udalski et al. (2001). The diagram is segregated into regions containing (a) blue objects, (b) AGB stars, (c) red supergiants, and (d) sub-TRGB red giants to aid in determining the stellar types of stars in the infrared color-magnitude diagram (Figure 4). The TRGB is located at $\mathrm{M}_{I}=-4$, in the center of the gap separating regions $\mathrm{b}$ and $\mathrm{d}$. 


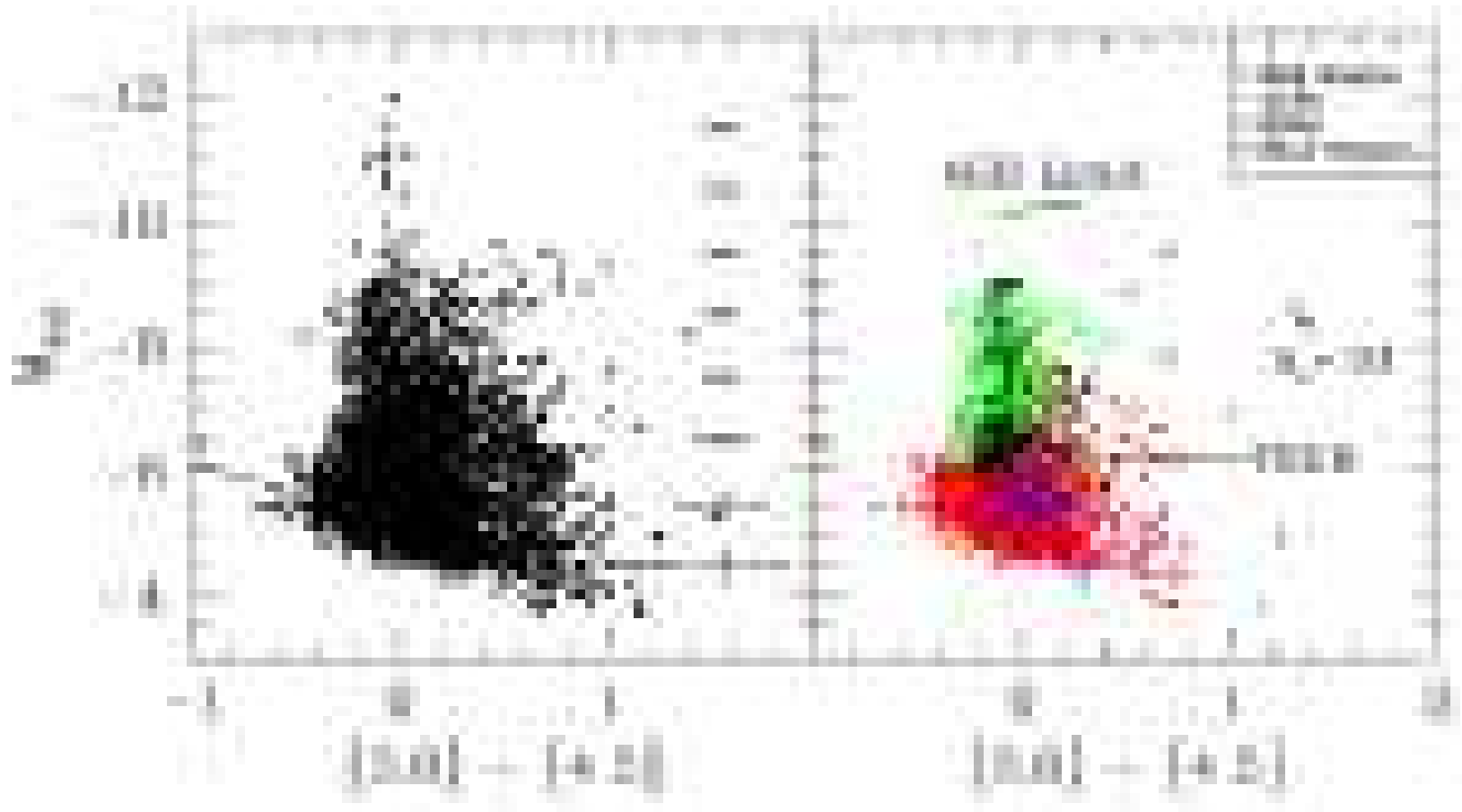

Fig. 4.- $3.6 \mu \mathrm{m}$ absolute magnitude versus [3.6]-[4.5] color magnitude diagram of IC 1613. The left panel shows all of the point sources detected at 3.6 and $4.5 \mu \mathrm{m}$. The right panel shows objects also detected in the Udalski et al. (2001) $V$ and $I$ data, segregated according to Figure 3 with sub-TRGB red giants as red squares, AGB stars as green triangles, RSGs as black stars, and blue objects as blue circles. Photometric 1- $\sigma$ errors, averaged over 1 mag bins, are shown on the right side of the left panel. The AGB limit, TRGB, and a vector showing 10 magnitudes of visual extinction are shown in the right panel. See $\$ 4$ for discussion. 


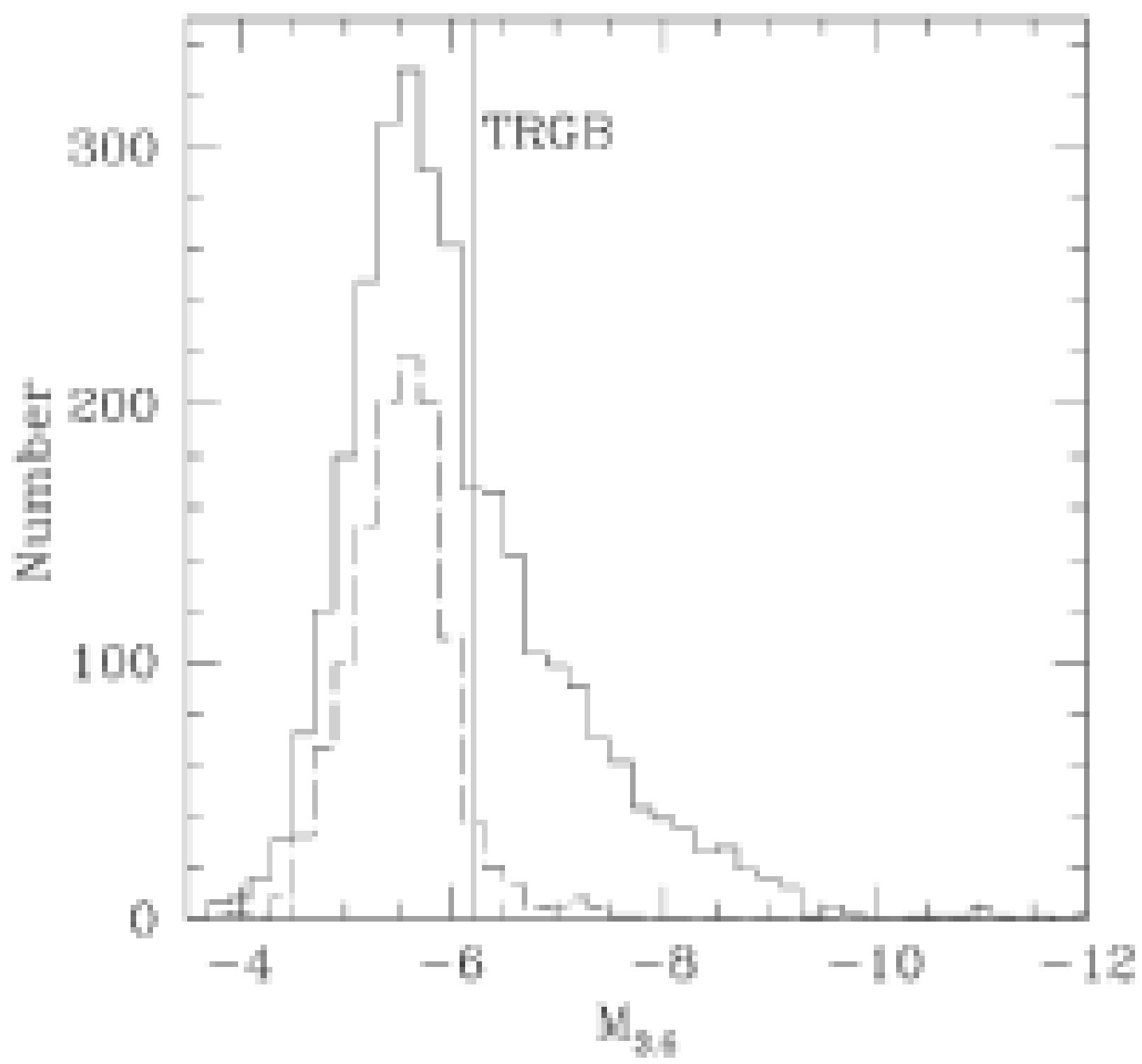

Fig. 5.- The $3.6 \mu \mathrm{m}$ luminosity functions for all of the stars in IC 1613 that were detected at 3.6 and $4.5 \mu \mathrm{m}$ (solid line) and for those detected at 3.6 and $4.5 \mu \mathrm{m}$ and optically classified as sub-TRGB red giants (dashed line). The value we adopt for the $3.6 \mu \mathrm{m}$ TRGB is shown at $\mathrm{M}_{3.6}=-6.2$ (see $\$$ for discussion). 


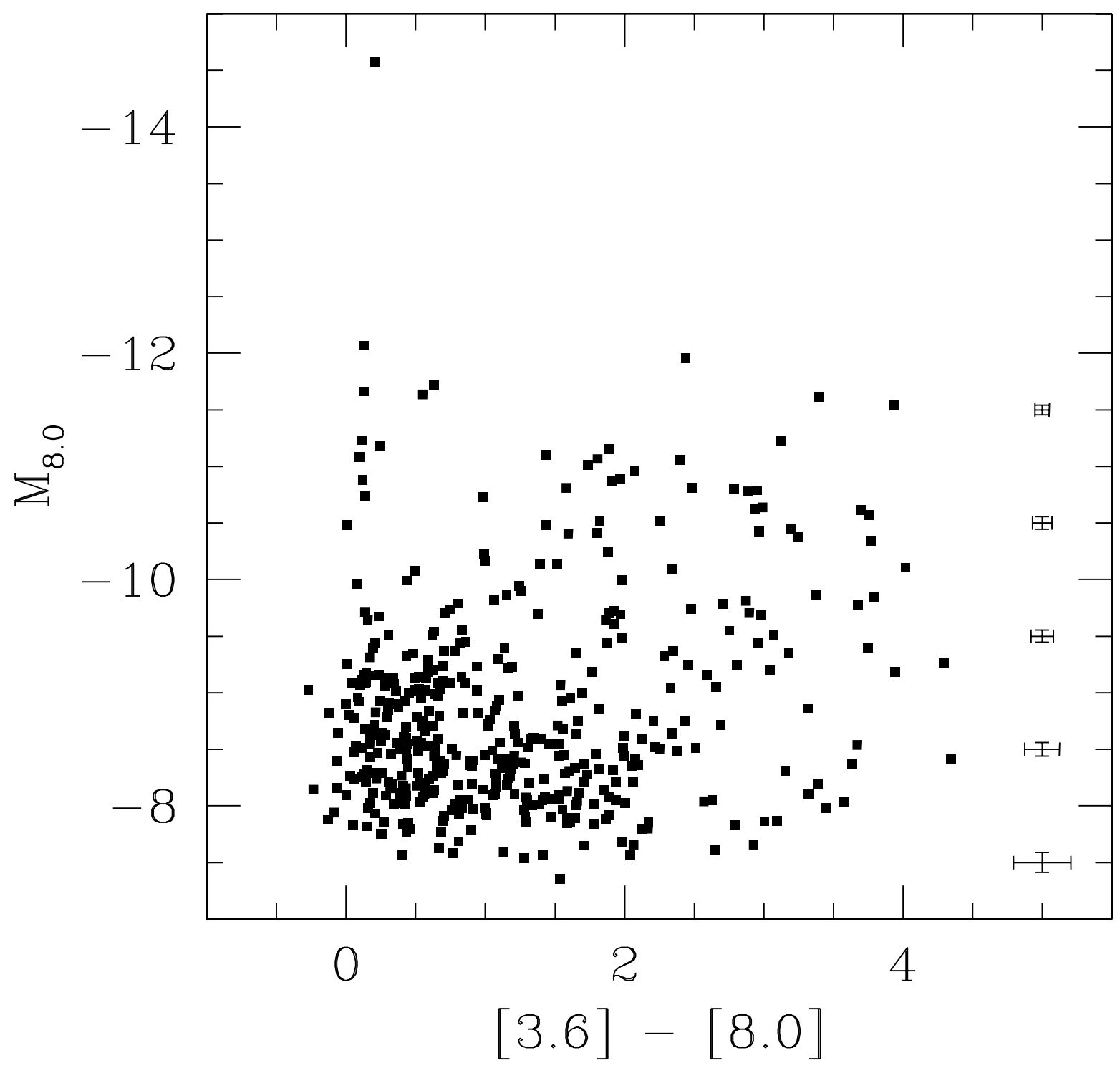

Fig. 6.- The $8.0 \mu \mathrm{m}$ absolute magnitude versus [3.6]-[8.0] color magnitude diagram of IC 1613. Photometric 1- $\sigma$ errors, averaged over 1 mag bins are shown on the right. 


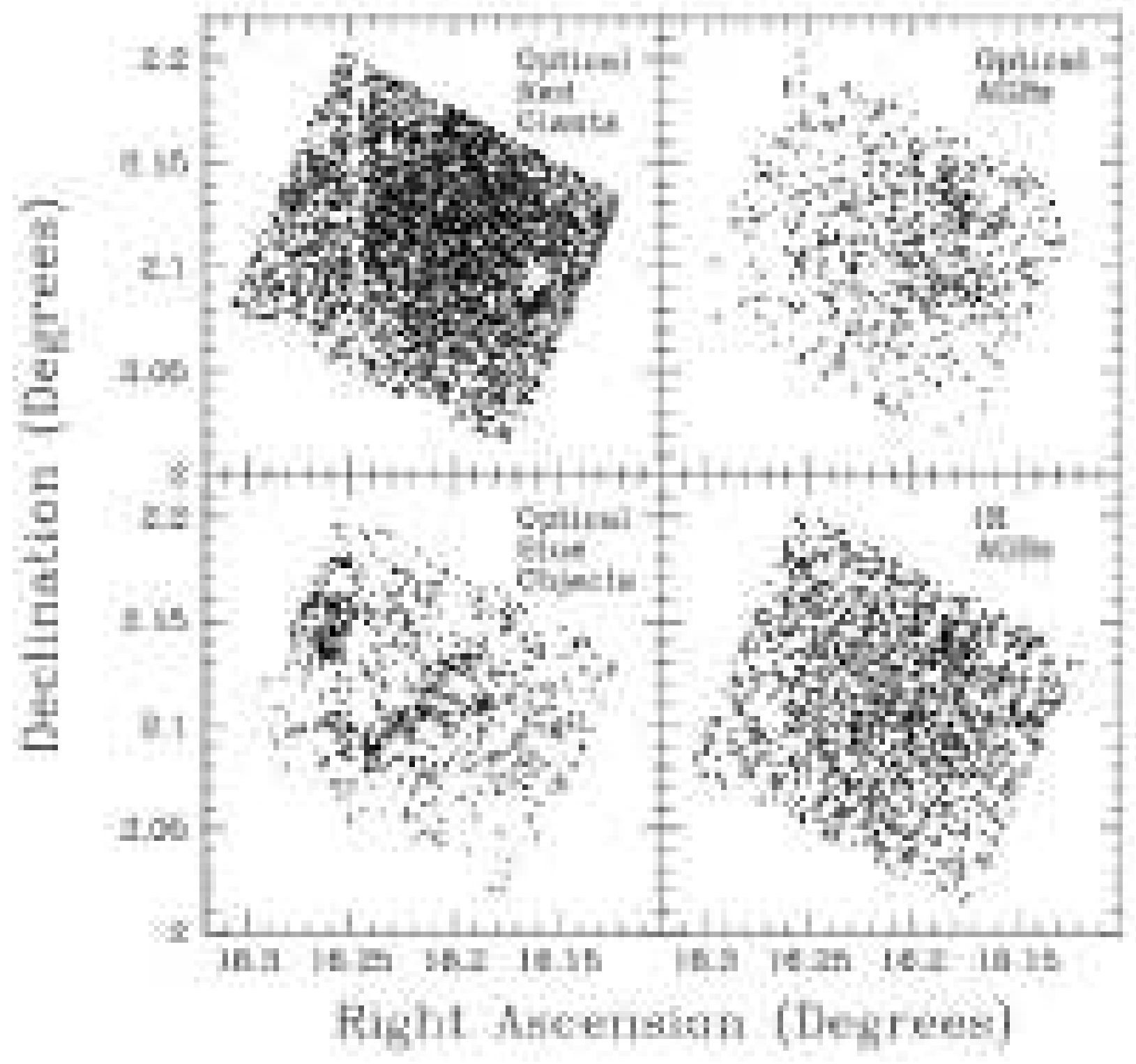

Fig. 7.- Positions of stars according to their optical classification, with the exception of the bottom right panel, which shows the positions of AGB stars detected in the IR. 


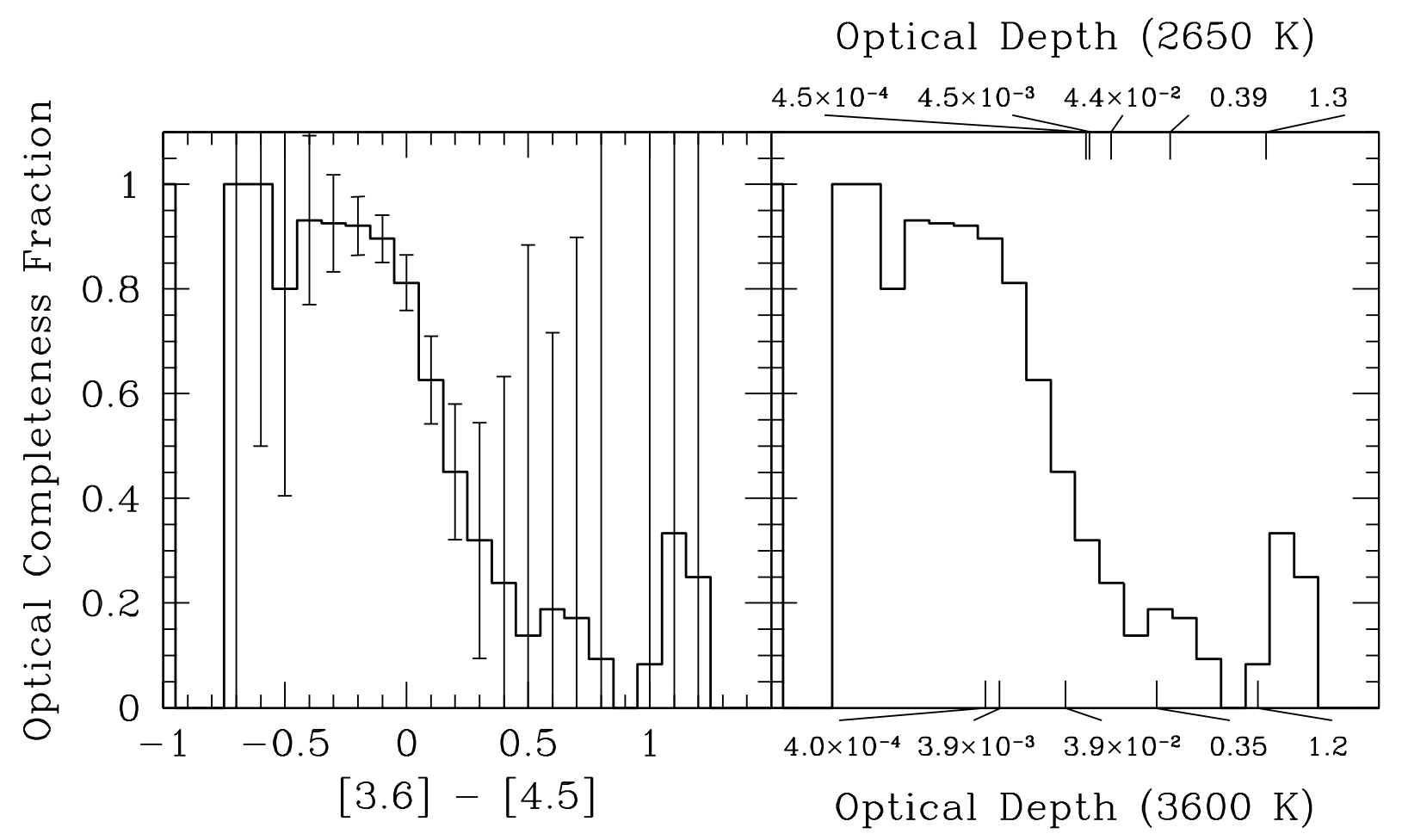

Fig. 8.- Fraction of objects more luminous than the TRGB at $3.6 \mu \mathrm{m}$ that are also detected in the Udalski et al. (2001) $V$ and $I$ photometry as a function of [3.6]-[4.5] color (left panel) and as a function of wind optical depth assuming AGB stars with wind composition of $85 \%$ $\mathrm{AMC}+15 \% \mathrm{SiC}$ and effective temperatures of $2650 \mathrm{~K}$ (top axis) and $3600 \mathrm{~K}$ (bottom axis). See $\$ 5.3$ for a detailed discussion of mass-loss modeling. The error bars were created by taking the square root of the number of optically detected AGBs and dividing by the total number of AGBs seen in at 3.6 and $4.5 \mu \mathrm{m}$ and therefore only represent the degree to which small number statistics might affect the overall trend. 

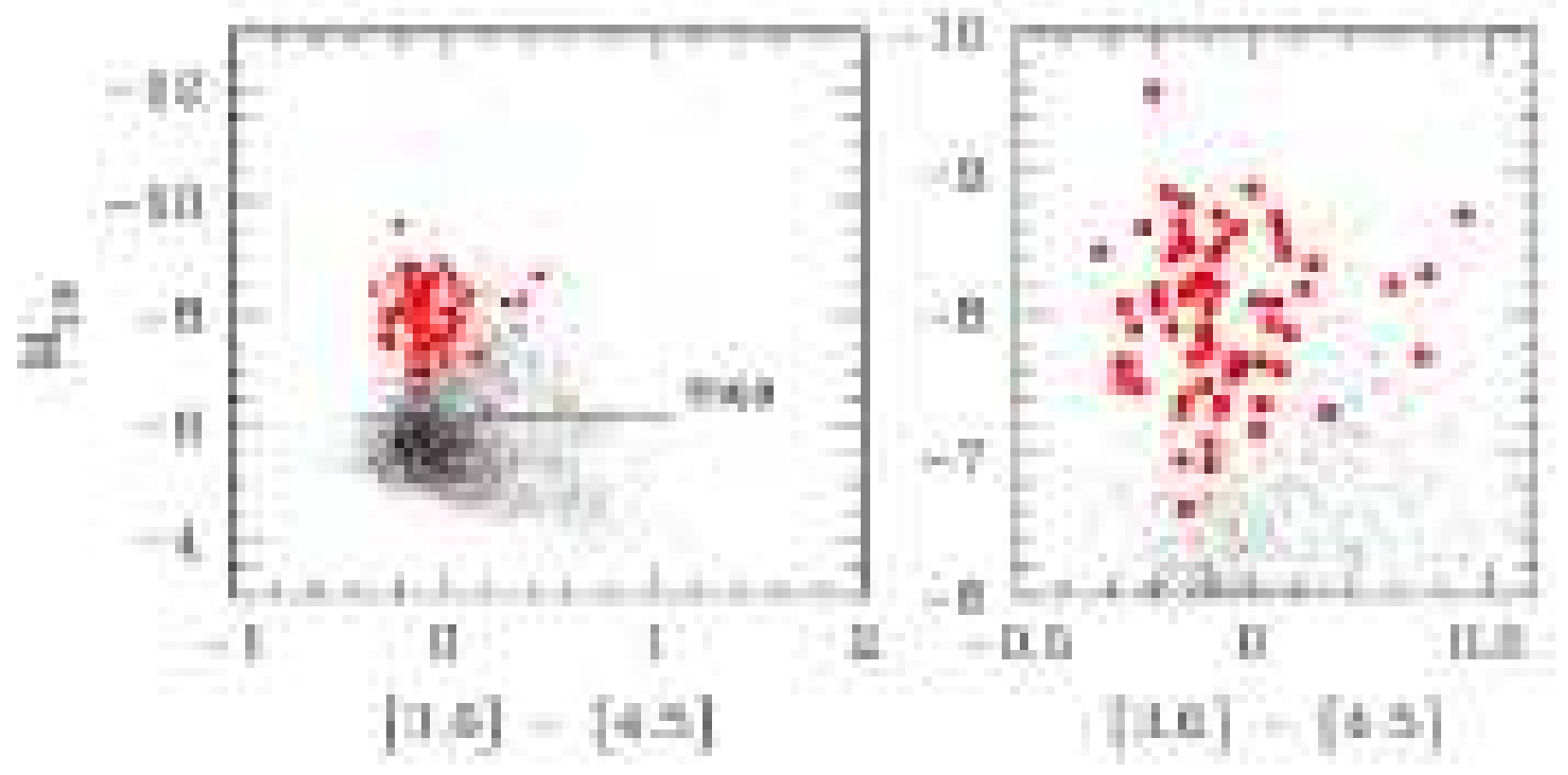

Fig. 9.- Absolute $3.6 \mu \mathrm{m}$ versus [3.6]-[4.5] color-magnitude diagram. The small black points are our IRAC detections and the large red circles are our measurements of the carbon stars identified by Albert et al. (2000). Note in particular the four carbon stars with $[3.6]-[4.5]>0.2$, which are likely losing significant mass. The right panel is magnified to help distinguish the points. 


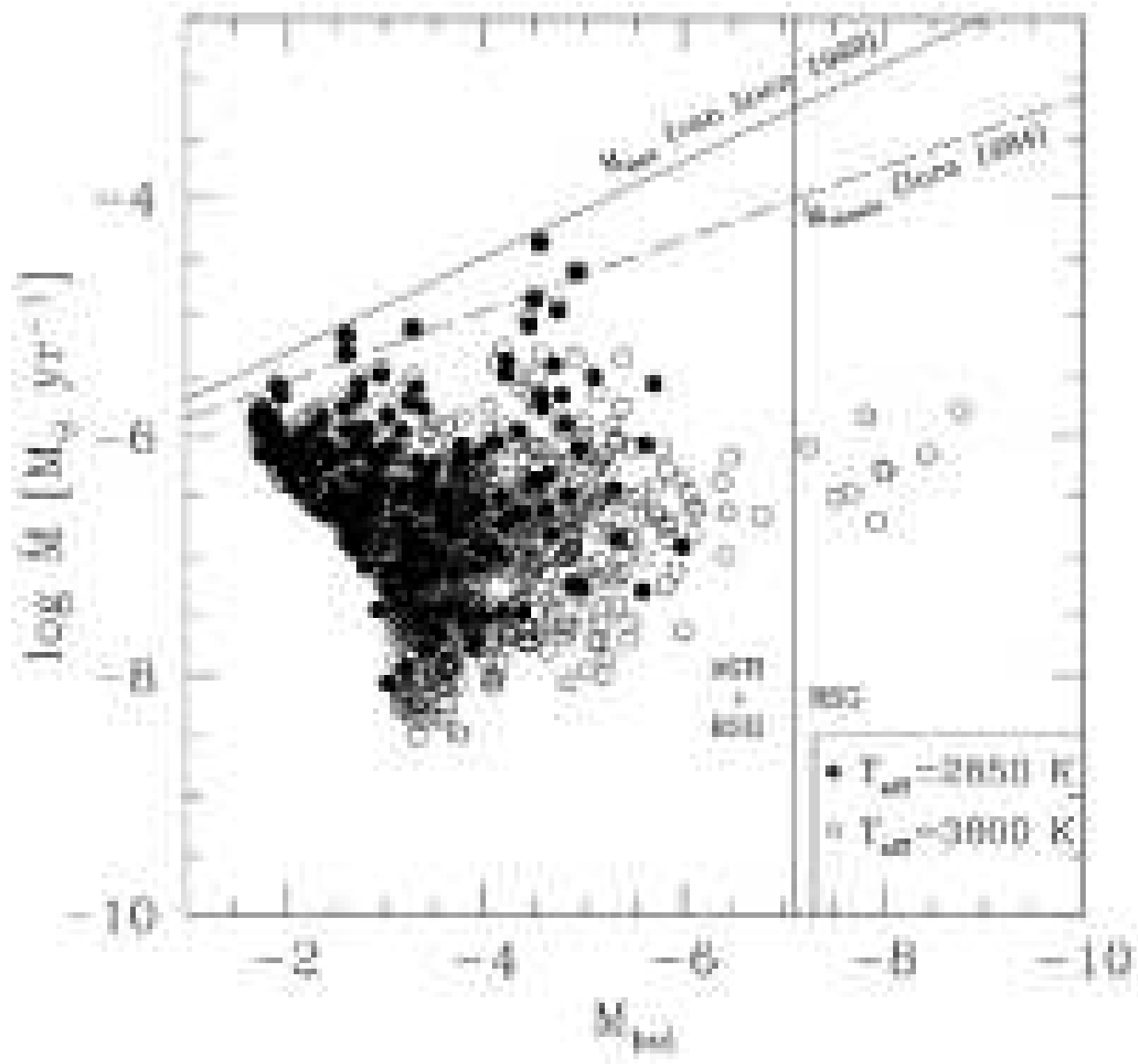

Fig. 10.- The mass-loss rate versus bolometric magnitude for all objects brighter than the the TRGB at $3.6 \mu \mathrm{m}$. The mass-loss rates assume a gas-to-dust ratio of $4.8 \times 10^{-4}$, a wind composition of $85 \% \mathrm{AMC}+15 \% \mathrm{SiC}$ and effective temperatures of $\mathrm{T}_{\text {eff }}=2650 \mathrm{~K}$ (filled circles) and $\mathrm{T}_{\text {eff }}=3600 \mathrm{~K}$ (open circles), using the mass loss prescription of van Loon (2000) (see \$5.3). The bottom dashed line is the Jura (1984) single-scattering mass-loss limit, and the top solid line is the empirical maximum mass-loss limit observed in the LMC (van Loon et al. 1999). The AGB limit is shown as a vertical line at $\mathrm{M}_{b o l}=-7.1$. 